\section{Polymer Density around a Sphere}

\section{R. Tuinier and H. N. W. Lekkerkerker*}

Van't Hoff Laboratory for Physical and Colloid Chemistry, Debye Research Institute, University of Utrecht,

Padualaan 8, NL-3584 CH Utrecht, The Netherlands

Received November 8, 2001

Revised Manuscript Received J anuary 17, 2002

The depletion of polymers near an interface is a phenomenon that determines the stability of colloidpolymer mixtures occurring in many systems. As an example, in food formulations the depletion of polysaccharides from the protein surface is a common situation, ${ }^{1-7}$ determining the (in)stability of such products. The onset of phase separation is an item that directly relates to consumer acceptance. In the past the focus has mainly been on either the "colloid limit", ${ }^{8-11}$ where the polymers are relatively small as compared to the spheres, or on the "protein limit", ${ }^{12-15}$ where the sphere size is negligible with respect to the characteristic polymer length scale. The final aim is to understand the depletion interaction for arbitrary polymer-sphere size ratio with the excluded-volume interaction of the polymer segments taken into account. To be able to do so, the polymer density around a single sphere for arbitrary size ratio is a quantity of primary importance. In this note we propose an equation that describes the polymer concentration profile very well as a function of the polymer-sphere size ratio.

The polymer density around a single sphere can be obtained if the (nonlinear differential) Edwards-de Gennes diffusion equation ${ }^{16-19}$

$$
\frac{\xi^{2}}{2} \nabla^{2} g=g^{3}-g
$$

is solved, where $\xi$ is the correlation length, with boundary conditions $\mathrm{g}=0$ at the sphere surface and $\mathrm{g}=1 \mathrm{far}$ from the sphere. The quantity $\mathrm{g}$ is defined as $\mathrm{g}^{2}=\mathrm{n}(\mathbf{r}) /$ $n_{b}$, where $n(r)$ is the segment concentration at position $\mathbf{r}$ and $n_{b}$ is the bulk segment concentration. No general analytical solution for arbitrary size ratio $\xi / \mathrm{R}$, where $\mathrm{R}$ is the sphere radius, has been found for this differential equation yet. Odijk ${ }^{13}$ solved the profile around a single hard sphere for the case $\mathrm{R} \ll \xi$ and found

$$
g=\frac{x}{R+x}
$$

where $x$ is the distance from the sphere surface. This result is compared with numerical solutions to eq 1 in Figure 1 for various size ratios $\xi / R$ in the small sphere range: $\xi / R=10,30$, and 100 . The symbols are the results of the numerical solution to eq 1 (see ref 15), and the dashed curves are the results of eq 2 . For $\xi=$ $100 R$ the agreement is satisfying, indicating that Odijk's limit can be used for small spheres. For the size ratio $\xi / \mathrm{R}=30$ and for 10 the deviation of eq 3 from the numerical result is however significant.

For $\xi \ll R$, the lowest order in curvature expansion of eq 1 was solved by Maassen, Eisenriegler, and Bringer. ${ }^{20}$ This leads to

\footnotetext{
* Corresponding author. e-mail: h.n.w.lekkerkerker@chem.uu.nl.
}

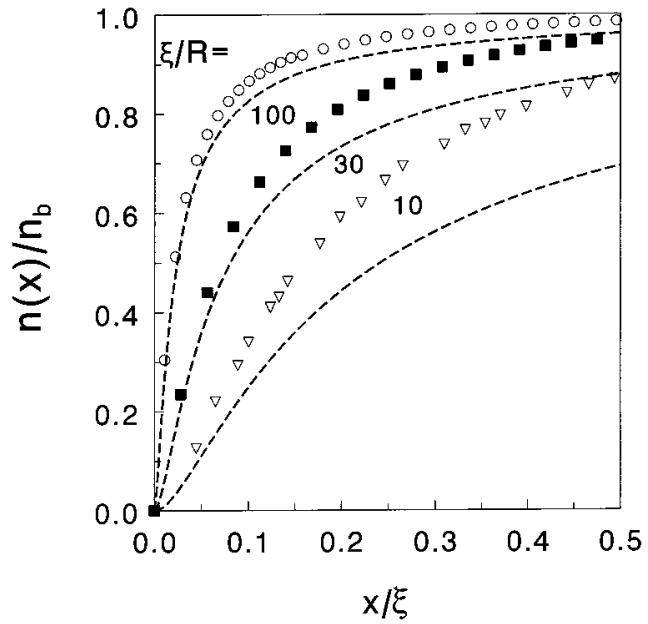

Figure 1. Concentration profile of polymer segments for various polymer - sphere size ratios in the small sphere regime. Dashed curves are given by eq 2, and the symbols are numerical results to eq 1 .

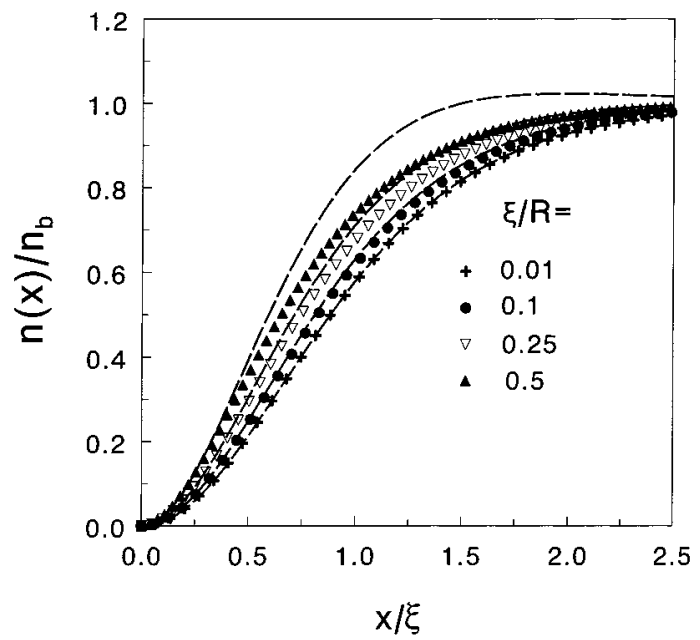

Figure 2. Polymer segment concentration profile near a single sphere for $\xi / \mathrm{R}$ ratios of $0.01,0.1,0.25$, and 0.5 as indicated. The data points are numerical solutions to eq 1 , and dashed curves follow eq 3 of Maassen, Eisenriegler, and Bringer (see Appendix $\mathrm{E}$ of ref 20).

$$
\frac{\mathrm{n}(\mathrm{x})}{\mathrm{n}_{\mathrm{b}}}=\tanh ^{2}\left(\frac{\mathrm{x}}{\xi}\right)+\frac{\xi}{\mathrm{R}} \mathrm{F}\left(\frac{\mathrm{x}}{\xi}\right)
$$

where

$$
\begin{array}{r}
F\left(\frac{x}{\xi}\right)=\frac{2}{3} \tanh \left(\frac{x}{\xi}\right)\left(\frac{3}{2} \frac{x / \xi}{\cosh ^{2}(x / \xi)}+\frac{2}{\cosh ^{2}(x / \xi)}+\right. \\
\left.\frac{3}{2} \tanh \left(\frac{x}{\xi}\right)-\frac{3}{2}-\frac{1}{2} \exp (-2 x / \xi)\right)
\end{array}
$$

In eq 3 the flat wall result $g=\tanh (x / \xi)$ of de Gennes (see ref 17 ) is recovered for $\xi / \mathrm{R} \rightarrow 0$. Numerical results (symbols) are given for $\xi / \mathrm{R}=0.01,0.1,0.25$, and 0.5 in Figure 2. Clearly, eq 3 (dashed curves) describes the trend well: an increase of $\xi / R$ moves the concentration profile toward the sphere surface. However, eq 3 overestimates the curvature effect already for $\xi / R$ values exceeding 0.3. The limiting cases, discussed above, are useful but do not cover the entire range of $\xi / \mathrm{R}$ ratios. 

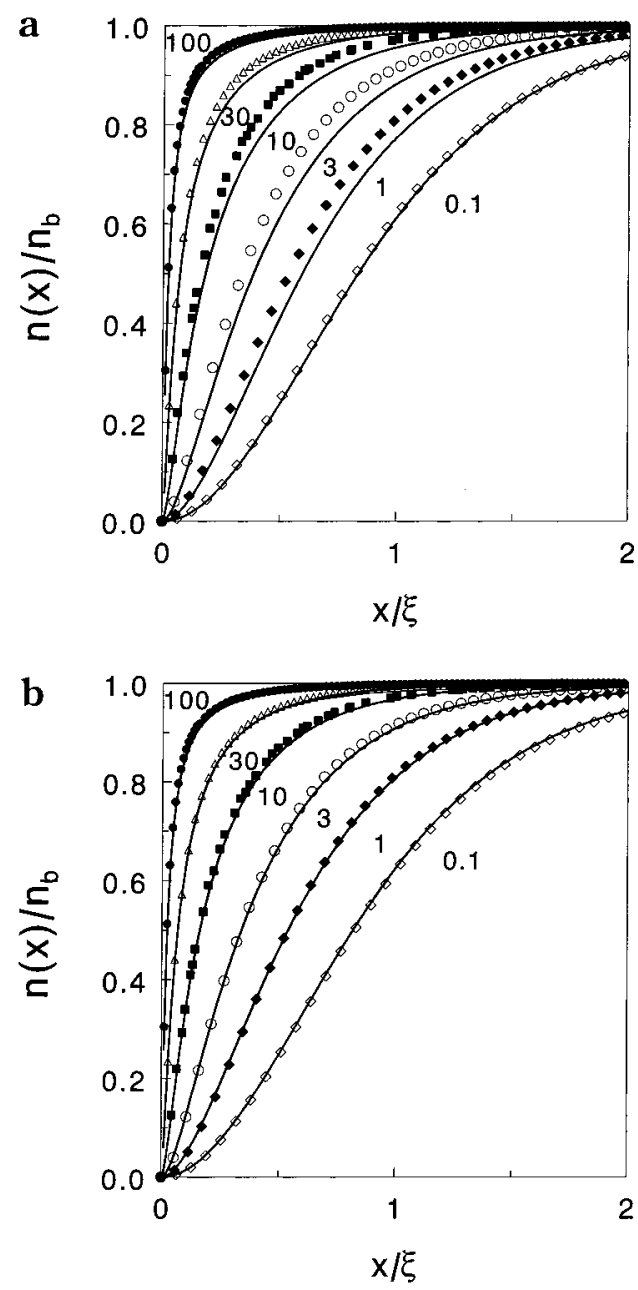

Figure 3. (a) Concentration profile of polymer segments for polymer-sphere size ratios $\xi / R=0.1,1,3,10,30$, and 100 as indicated. Equation 4 is given as the full curves, and the symbols are numerical results to eq 1 . (b) As (a) but the full curves are given by eq 6 .

Thus, the situation where the size ratio is of the order unity remains a problem.

By interpolating between depletion behavior near a small sphere, $g=x /(R=x)$, and the depletion near a large sphere, $g=\tanh (x / \xi)$, we propose

$$
g=\frac{x}{R+x}+\frac{R}{R+x} \tanh \left(\frac{x}{\xi}\right)
$$

In Figure 3a, eq 4 is plotted as the full curves and is compared with numerical solutions ${ }^{15}$ of eq 1 (symbols) for a wide range of $\xi / \mathrm{R}$ ratios as indicated in the plot. It follows that the simple approximation equation (4) works well but gives a slight underestimation of the polymer density for intermediate size ratios.

The form of the polymer density profile of ideal polymer segments by Taniguchi et al. ${ }^{21}$ (see al so ref 14), which applies for arbitrary size ratio, suggests a solution of the form

$$
g^{2}=\left(\frac{R}{R+x}\right)^{2}\left(\left(\frac{x}{R}\right)^{2}+2\left(\frac{x}{R}\right) A\left(\frac{x}{\xi}\right)+B\left(\frac{x}{\xi}\right)\right]
$$

with $B=\tanh ^{2}(x / \xi)$ and $A(x / \xi)$ an unknown function. From the numerical results it follows that $A(x / \xi)$ is independent of $R / \xi$; for various size ratios the numerical results of $A(x / \xi)$ follow a single curve. We found that $A=\tanh (x / \xi)$ (leading to eq 4) gives a slight underestimation of the numerical data while $A=1-$ $\exp (-2 x / \xi)$ gives a slight overestimation and that the mixed form $A=(1 / 3)[\tanh (x / \xi)+2(1-\exp (-2 x / \xi))]$ provides an extremely accurate solution for $\mathrm{g}$ :

$g=\left(\frac{x}{R+x}\right) \times$

$\sqrt{1+\frac{R}{x}\left(\frac{2}{3} \tanh \left(\frac{x}{\xi}\right)+\frac{4}{3}\left[1-\exp \left(-\frac{2 x}{\xi}\right)\right]\right)+\left(\frac{R}{x}\right)^{2} \tanh ^{2}\left(\frac{x}{\xi}\right)}$

The accuracy of eq 6 is illustrated in Figure 3b, where eq 6 is compared (full curves) with numerical data from Figure $3 a$ (symbols).

Acknowledgment. The work presented was supported by the Council for Chemical Sciences of the Netherlands Organization for Scientific Research and Unilever Research Vlaardingen. H. M. Schaink is thanked for using his computer program for the numerical solution of eq 1. G. J. Fleer and P. Van der Schoot are acknowledged for inspiring us to investigate this problem at an early stage, and we are grateful to J. K. G. Dhont, G. J . Vroege, M. E. Cates, and M. Fuchs for useful discussions.

\section{References and Notes}

(1) Hoskins, R.; Robb, I. D.; Williams, P. A.; Warren, P. B. J . Chem. Soc., Faraday Trans. 1996, 92, 4515.

(2) Grinberg, V. Ya.; Tolstoguzov, V. B. Food Hydrocolloids 1997, 11, 145.

(3) Syrbe, A.; Bauer, W. J .; Klostermeyer, H. Int. Dairy J . 1998, 8, 179.

(4) Tuinier, R.; Ten Grotenhuis, E.; Holt, C.; Timmins, P. A.; De Kruif, C. G. Phys. Rev. E 1999, 60, 848.

(5) Tuinier, R.; de Kruif, C. G. J . Chem. Phys. 1999, 110, 9296.

(6) Tuinier, R.; Dhont, J . K. G.; De Kruif, C. G. Langmuir 2000, 16, 1497.

(7) Doublier, J .-L.; Garnier, C.; Renard, C.; Sanchez, C. Curr. Opin. Colloid Interface Sci. 2000, 5, 184.

(8) Asakura, S.; Oosawa, F. J . Chem. Phys. 1954, 22, 1255.

(9) Vrij, A. Pure Appl. Chem. 1976, 48, 471.

(10) Lekkerkerker, H. N. W.; Poon, W. C. K.; Pusey, P. N.; Stroobants, A.; Warren, P. B. Europhys. Lett. 1992, 20, 559.

(11) Ilett, S. M.; Orrock, A.; Poon, W. C. K.; Pusey, P. N. Phys. Rev. E 1995, 51, 1344.

(12) de Gennes, P. G. C. R. Acad. Sci. B 1979, 288, 359

(13) Odijk, T. Macromol ecules 1996, 29, 1842.

(14) Eisenriegler, E.; Hanke, A.; Dietrich, S. Phys. Rev. E 1996, 54, 1134.

(15) Schaink, H. M.; Smit, J . A. M. J . Chem. Phys. 1997, 107, 1004.

(16) Edwards, S. F.; Freed, K. F. J . Phys. A 1969, 2, 145

(17) de Gennes, P. G. Scaling Concepts in Polymer Physics; Cornell University Press: I thaca, NY, 1979; Chapter 9.

(18) Grosberg, A. Y.; Khoklov, A. R. Statistical Mechanics of Macromolecules; AIP: New York, 1994.

(19) Fleer, G. J .; Cohen Stuart, M. A.; Scheutjens, J . M. H. M.; Cosgrove, T.; Vincent, B. Polymers at I nterfaces; Chapman \& Hall: London, 1993; Chapter 4.

(20) Maassen, R.; Eisenriegler, E.; Bringer, A. J . Chem. Phys. 2001, 115, 5292

(21) Taniguchi, T.; Kawakatsu, T.; Kawasaki, K. In Slow Dynamics in Condensed Matter; AIP Series; Kawasaki, K., Ed.; 1992, 256, 503.

MA0119474 